\title{
Un capteur d'extensométrie miniature à deux voies
}

\author{
Jean-Charles FERRAND \\ I.N.R.A., Station de Recherches sur la Qualité des Bois \\ Centre de Recherches forestières de Nancy, \\ Champenoux, F 54280 Seichamps
}

\begin{abstract}
Résumé
Un capteur d'extensométrie miniature à deux voies a été réalisé. Il permet de mesurer les déformations dans deux directions perpendiculaires, et sa sensibilité est de 5 microdéformations. Il s'applique sur le bois sans collage.
\end{abstract}

Après avoir étudié une méthode indirecte de mesure de la déformation potentielle longitudinale $\varepsilon_{\mathrm{L}}$ à la périphérie cies trones, FERrand (1982) a montré le besoin d'un capteur extensométrique à deux voies pour la mesure des déformations $\varepsilon_{L_{r}}$ et $\varepsilon_{\mathrm{T}}$ dues aux contraintes de croissance longitudinalle et tangentielle des arbres forestiers. Pour être pratique, un tel capteur devait répondre aux exigences suivantes :

1) fournir une mesure fidèle dans deux directions perpendiculaires (longitudinale et tangenticlle sur l'arbre),

2) ne pas nécessiter de collage sur le bois pour accélérer le déroulement des mesures,

3) ĉtre adlapté à toutes les surfaces, et pas seulement aux surfaces quasi planes,

4) avoir des dimensions très faibles pour minimiser le traumatisme infligé à l'arbre et permettre les mesures sur des tiges de diamètre faible,

5) comporter sur chaque lame quatre jauges câblées en pont complet afin d'assurer unc bonne stabilité de la mesure dans toutes les conditions de température.

Les exigenees 2 et 3 aboutissent fatalement à une configuration où l'appareil ne repose sur le bois que par trois pointes. Un capteur à 8 pointes rigides soudées sur des lames déformables a été proposé par Seichepine (1980) pour des mesures sur échantillons rabotés. Il a montré que les pointes ne glissent pas sur le bois. Par conséquent, il est possible de concevoir un extensomètre reposant sur le bois par des pointes et fonctionnant correctement.

Le capteur proposé est présenté sur la photo 1 ; il comporte un corps rigide $\mathbf{A}$ en forme d'équerre, un pied rigide $B$, et deux pièces de protection $C$ (figure 1) qui servent également à la fixation des lames déformablies sur lesquelles sont collées les jauges de mesure (figure 2). Le pied $B$ et les lames se terminent en pointe. La force d'appui est assurée, soit par un ruban élastique fixé sur le tronc avec des punaises, 
soit, comme sur la photo 1, par une potence munie à sa base d'une pointe d'acier qu'on enfonce dians le bois et qui appuie sur le corps du capteur par l'intermédiaire d'un ressort. Afin de ne pas glisser, les lames doivent être minces pour ne pas résister trop aux déplacements que leur impose le bois, et se terminer par une pointe rigide mais suffisamment aiguë. Cela va à l'encontre d'une sensibilitité importante puisque celle-ci augmente avec l'épaisseur de la lame. Finalement, on a découpé les lames dans une tôle d'acier écroui de $0,15 \mathrm{~mm}$ d'épaisseur. Toutes les arêtes ont été usinées en plaçant la tôle entre des pièces de laiton. La réalisation de ce travail délicat est due à J.R. Perrin (*) qui doit être chaleureusement remercié ici.
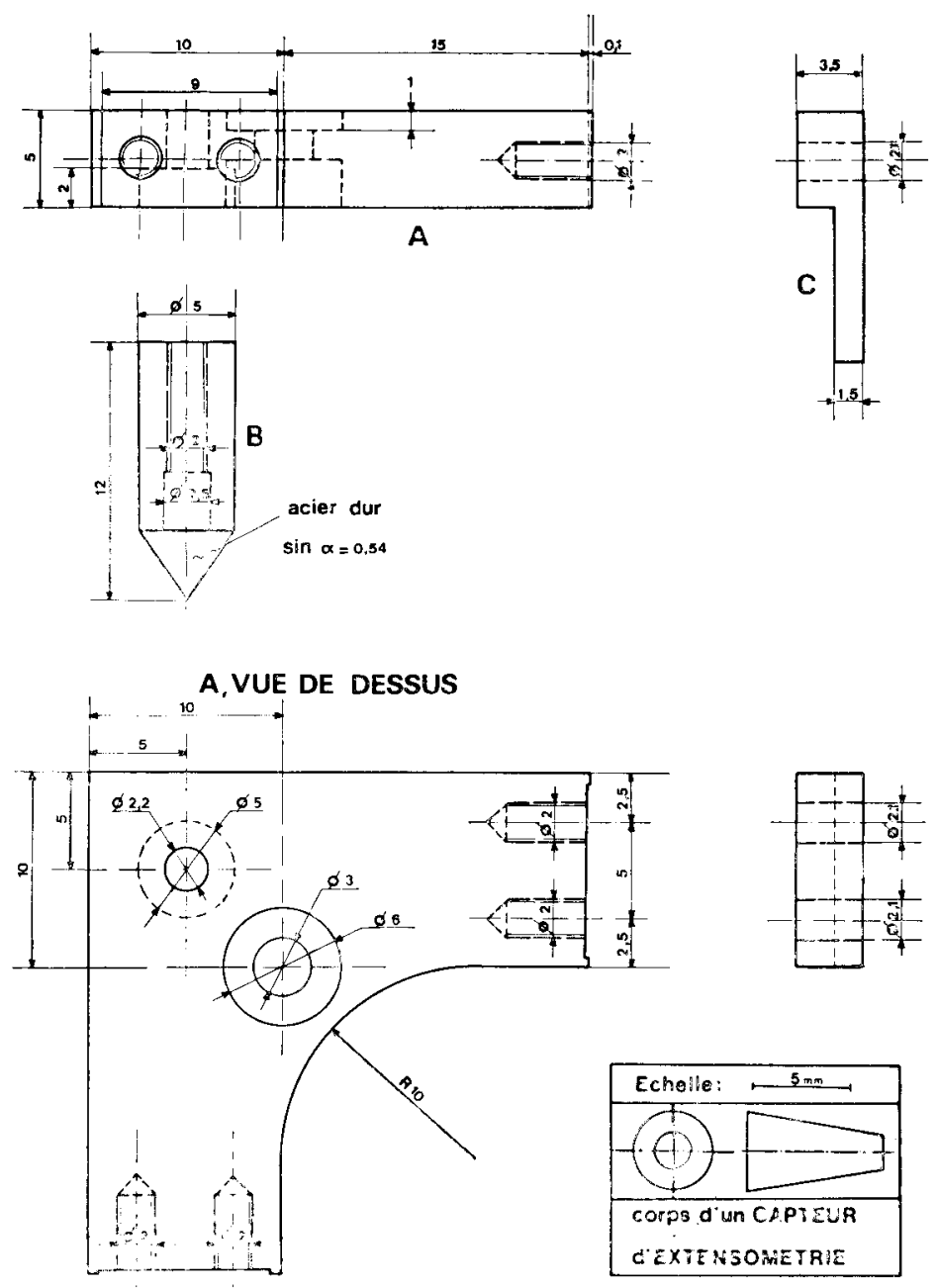

FIgURE 1

Capteur d'extensométrie, corps

Extensometric sensor, body

(*) Station de Recherches sur la Qualité des Bois. 


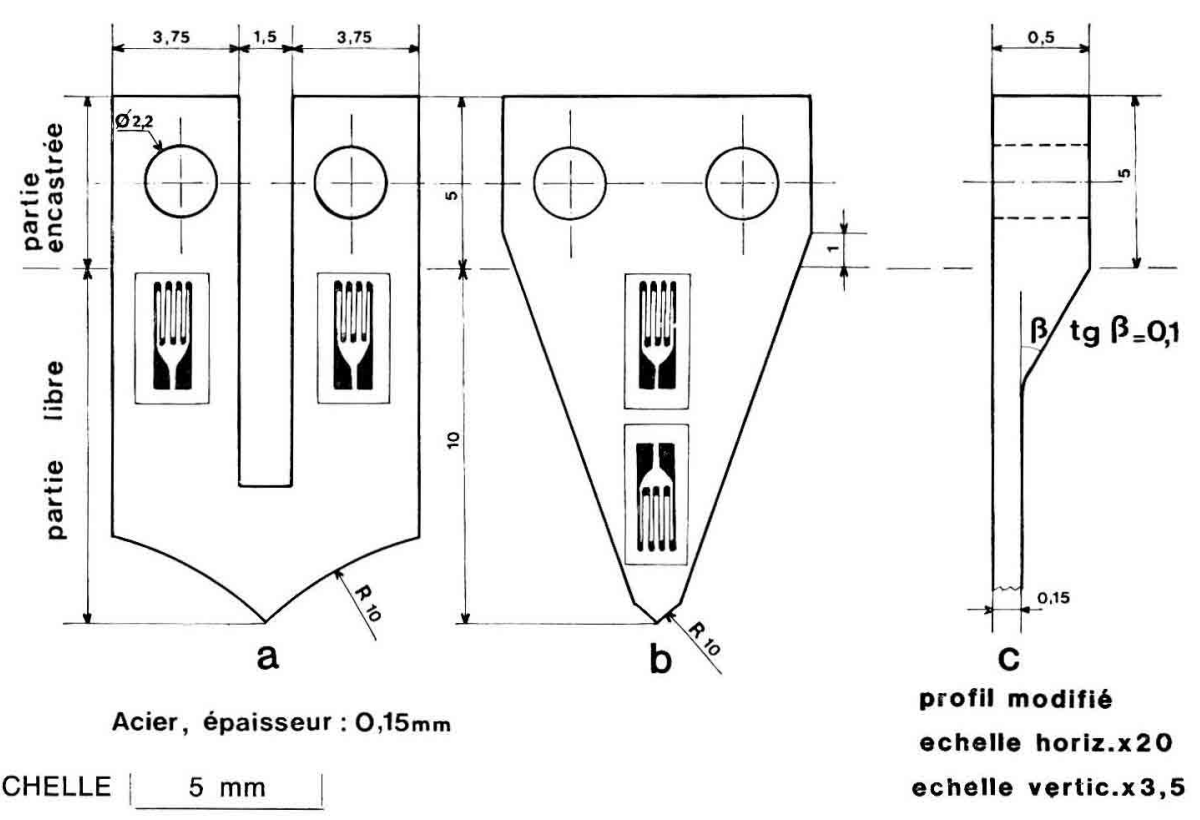

Figure 2

Capteur d'extensométrie, lames («corps d'épreuve») et emplacement des jauges Extensometric sensor, blades and location of the gages

Les calculs classiques de la résistance des matériaux montrent que, pour les dimensions choisies $(l=20 \mathrm{~mm})$ entre la pointe fixe et les extrémités des lames mobiles; $\mathrm{L}=10 \mathrm{~mm}$ pour la longucur libre de ces lames et $\mathrm{b}=0,15 \mathrm{~mm}$ pour Icur épaisseur (figure 2 a) la déformation relative maximale des lames $\left(\varepsilon_{1}\right)$ est d'environ 0,045 fois celle du bois entre les pointes $(\varepsilon)$ :

$$
\left[\frac{\varepsilon_{1}}{\varepsilon}=\frac{3}{2} \frac{\mathrm{b} /}{\mathrm{L}^{2}}\right]
$$

En disposant dieux jauges sur chaque face, câblées en pont complet, on multiplie le signal par 4 . Cependant, on ne peut coller les jauges à l'endroit où la déformation est maximale, c'est-à-dire au ras de lencastrement de la lame dans le corps, mais seulement à $1 \mathrm{~mm}$ de celui-ci. La déformation en un point de la lame rectangulaire étant proportionnelle à sa distance à la pointe, on obtient finalement un gain théorique qui vaut : $K=0,045 \times 4 \times 0,9=0,162$. La déformation relative minimale enregistrée par le pont de mesure étant de $10^{-6}$ s.d. la sensibilité théorique du capteur sera done d'environ 6 microdéformations ( 1 microdéformation $=10^{-6}$ s.d.).

La figure 3 montre le résultat de l'étalonnage d'une voie du capteur sur une poutre en bois de peuplier de dimensions $1,5 \times 5,5 \times 100 \mathrm{~cm}$, déformée en flexion 
circulaire. La linéarité est excellente, et l'accord est tout aussi bon avec les indications fournies par des jauges collées sur la poutre. Le gain réel $K_{1}$ est de 0,208 , c'est-à-dire que la sensibilité est de 1 microdéformation pour 5 au lieu de 1 pour 6 comme dans le calicul théorique. Cette légère différence n'a rien d’alarmant et s'explique en grande partie par l'épaisseur dies joints de colle entre la lame et la trame de la jauge.

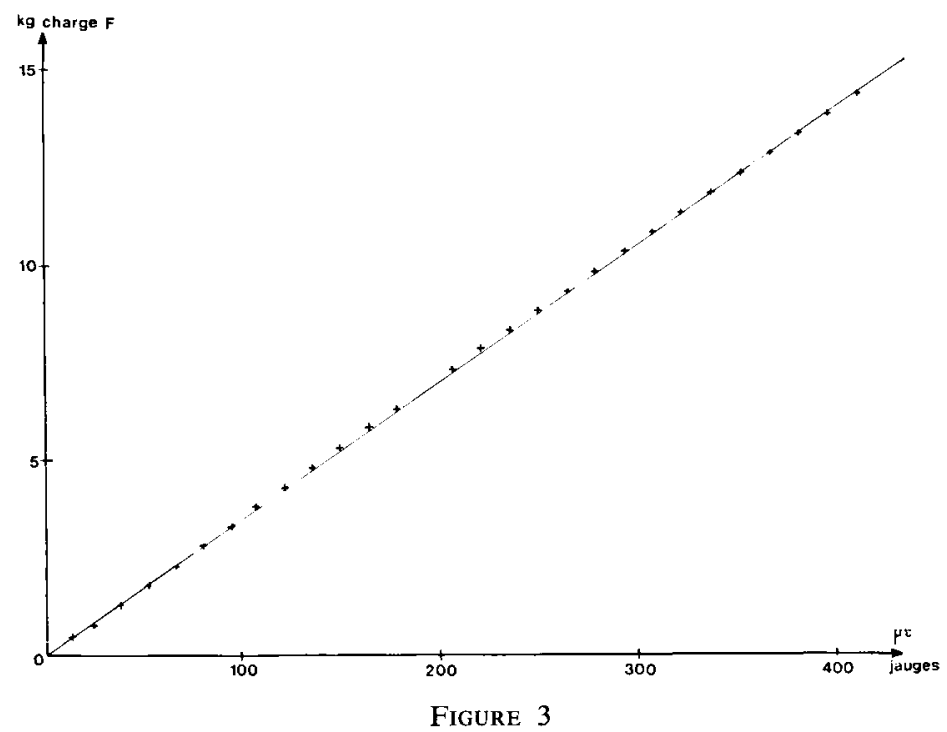

Etalonnage d'une voie du capteur sur une poutre de peuplier Calibration of one way of the sensor, on a timber beam (poplar)

La figure 3 montre qu'il n'y a pas de phénomène de glissement des pointes sur le bois. Mais on peut s'en assurer d'une autre façon, en calculant la force appliquée à la pointe d"une lame. Celle-ci vaut :

$$
\mathrm{f}=\frac{3 l}{\mathrm{~L}^{3}} \varepsilon \mathrm{E} \frac{\mathrm{ab^{3 }}}{12}
$$

(b, L, $l$ et $\varepsilon$ ont été définis ci-clessus); a est la largeur de la lame ; $\mathrm{E}$ est son module d'Young. On voit sur la figure $2 \mathrm{~B}$ que la largeur de la lame a été diminuée par une profonde encoche, précisáment pour réduire la force f. En appliquant cette formule avec un module d'Young de $211^{10}$ Pascal, on trouve qu'une déformation du bois $\varepsilon=210^{-3}$ s.d. produit une force denviron 0,050 Newton.

Le tableau 1 contient les résultats d'une manipulation au cours de laquelle on a suspendu au bout de la lame du captcur des masses étalon. Le capteur indique l'équivalient d'une déformation $\varepsilon=1,98 \quad 10^{-3}$ pour une masse de 5,5 grammes. L'accord avec la valeur calleulée est donc bon. De plus, il se maintient lorsque la déformation augmente puisque le signal fourni pour une charge de $10 \mathrm{~g}$ est exactement Ie double de celui obtenu avec $5 \mathrm{~g}$. 


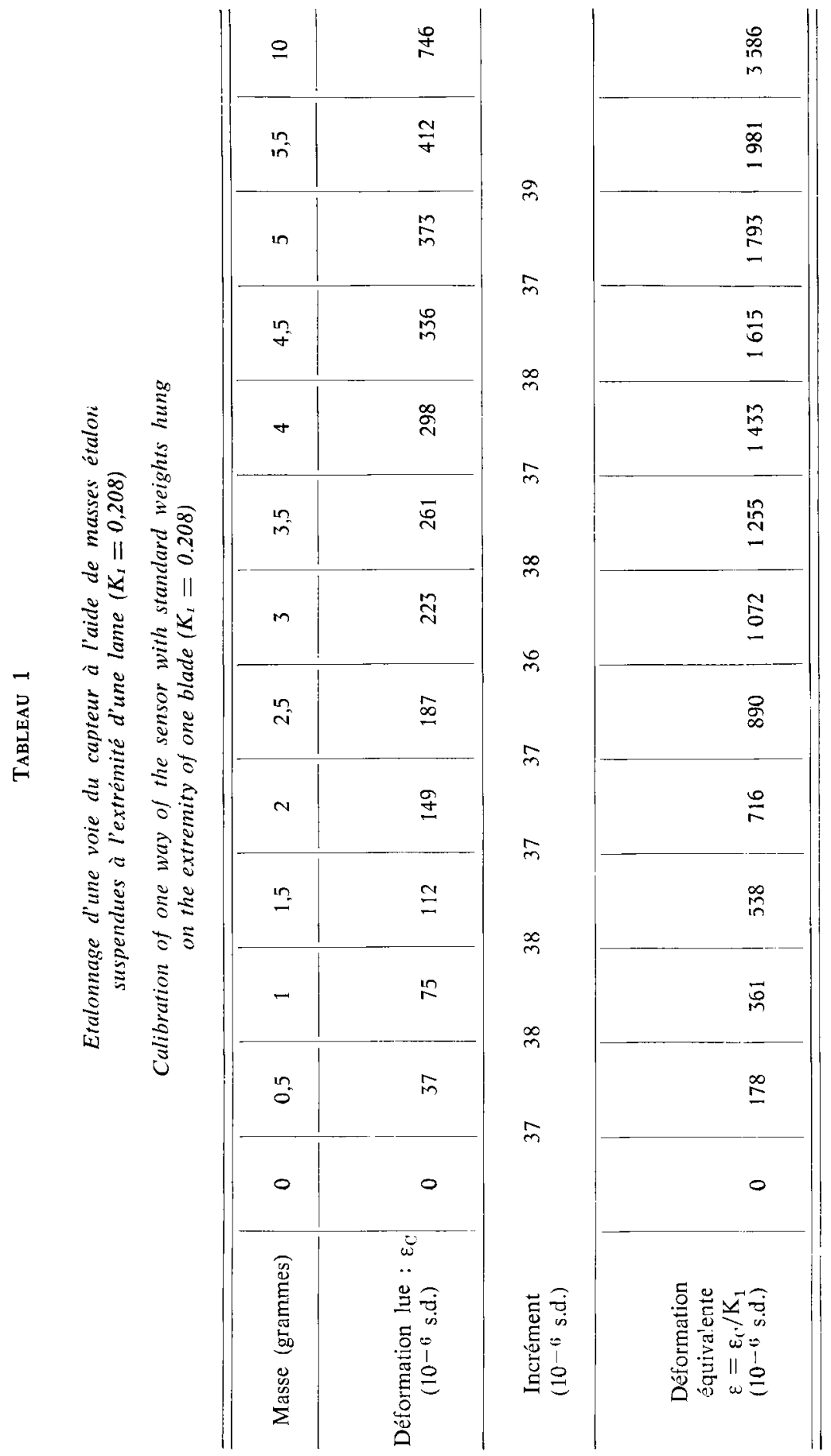


Ainsi, la petitesse des efforts mis en jeu, attestée tant par le calcul que par les résultats expérimentaux, est une garantie du bon fonctionnement du capteur, et de non glissement des lames.

\section{Note}

La figure 2 représente deux modifications possibles des lames du capteur.

La première (b) consiste à adopter une forme triangulaire. On obtient alors une déformation constante tout au long de la lame, ce qui permet de coller les jauges n'importe où sur l'axe de symétrie. Par rapport à la forme rectangulaire, et pour des dimensions identiques, la force $\mathbf{f}$ est plus faible, mais le gain $\mathbf{K}$ aussi.

La seconde modification (c) consiste à usiner des lames rectangulaires dont la partie déformable aurait les mêmes dimensions que les précédentes, mais qui seraient plus épaisses à proximité de l'encastrement: cette partie plus épaisse ne se déformerait presque pas, et les paramètres $\mathrm{K}$ et $\mathrm{f}$ seraient donc très peu modifiés. En revanche, on pourrait coller les jauges en plaçant leurs connexions du côté de l'encastrement, et non plus vers l'extrémité libre de la lame; cela supprimerait les liaisons électriques "aériennes» que l'on voit sur la photo $\mathrm{n}^{\circ} 1$, et qui risquent d'être détériorées lors du travail en forêt.

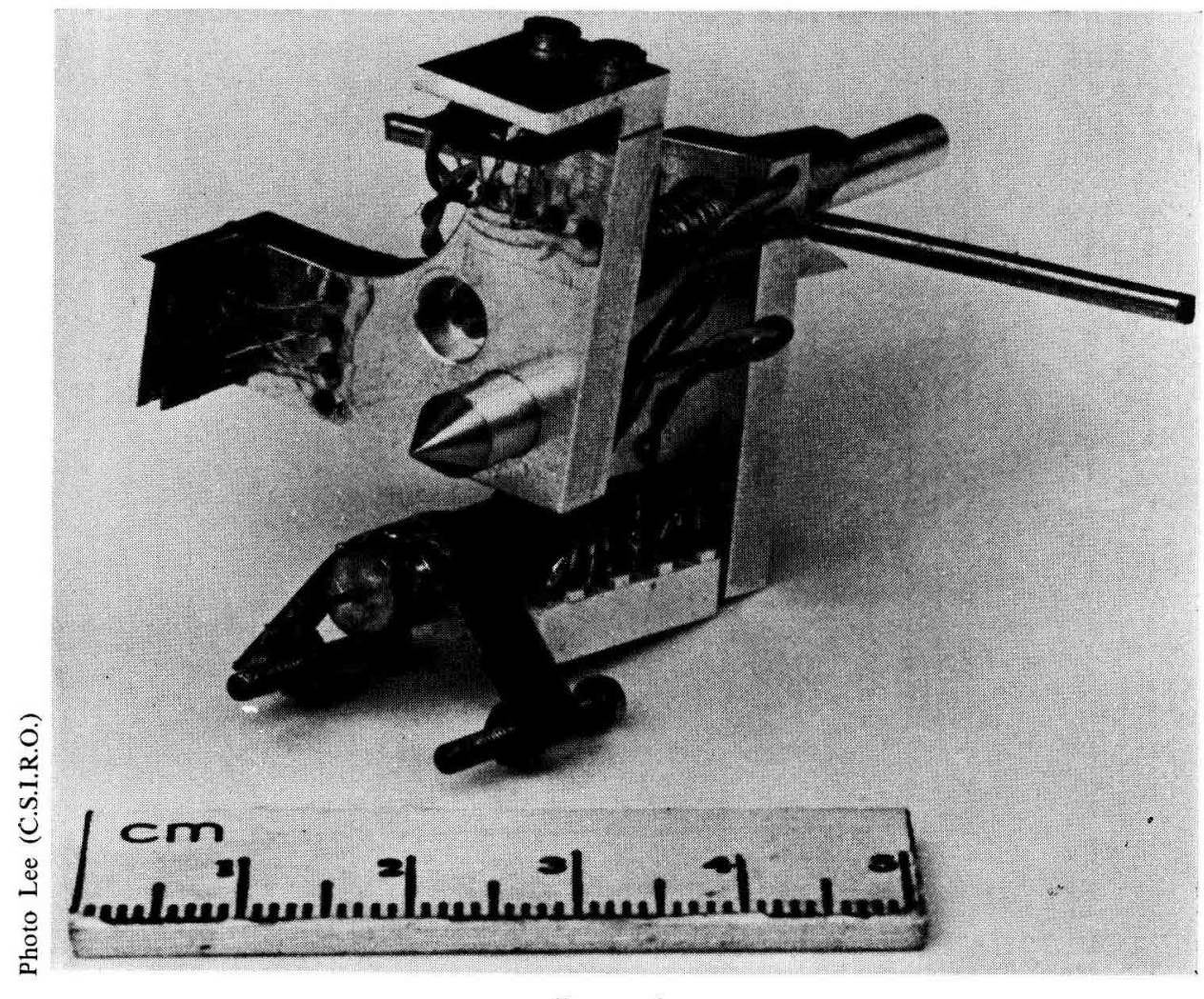

Рното 1

Prototype du capteur à deux voies réalisé selon les figures 1 et 2 a.

Le capteur est muni d'une potence destinée à l'appuyer sur l'arbre

Prototype of the extensometric sensor built accordingly to fig. 1 and 2 a. $A$ bracket is used to press the sensor on the tree 
Lorsque l'appareil est fixé sur un arbre, la relaxation des contraintes peut être obtenue soit en forant des trous à la mèche à bois, soit en perçant avec une scie à chaîne. Il est évident qu'un capteur extensométrique de ce type peut être utilisé non seulement en forêt, mais aussi en usine et au laboratoire pour une multitude d'usages.

Reçue pour publication le 7 juillet 1981.

\section{Summary}

\section{A miniature two-ways extensometric sensor}

A miniature two-ways extensometric sensor has been built. It allows the measurement of strains in two perpendicular directions and its sensitivity is 5 microstrains. It is used on timber without gluing.

\section{Références bibliographiques}

Ferrand J.Ch., 1982. Etude des contraintes de croissance. $1^{\text {re }}$ partie : Méthode de mesure sur carottes de sondage. Ann. Sci. for., 39 (2), 109-142.

Seichepine J.L., 1980. Mise au point d'une méthode expérimentale destinée à l'identification de la matrice des complaisances élastiques de solides anisotropes : application aux matériaux bois. Thèse Docteur-Ingénieur, I.N.P.L., Nancy, 10 octobre. 